\title{
A CLOCK-CONTROLLED CONSTANT-FREQUENCY GENERATOR
}

\author{
By A. B. Lewis
}

\section{ABSTRACT}

A synchronous motor generator set is described in which the motor is forced to rotate in synchronism with signals from a standard clock circuit. This result is obtained by first running a specially wound motor synchronously from a 3-phase commercial power line. The field of this synchronous motor is then electrically rotated about the motor frame by an amount which exactly compensates for the departure of the frequency of the cmmercial power from true 60 cycles. This rotation of the motor field is produced by a rotary synchroscope which is in turn controlled by thyratron tubes, the grids of which are excited by a clockdriven tuning fork. The output of the generator is used to operate cycle counters, synchronous timers, or other light synchronous machinery.

The possibilities and limitations of the machine are discussed and data are given to indicate the accuracy ( \pm 0.004 second) which may be expected from the machine when used as a timing device. Safety devices are described which shut down the machine should it for any reason fall out of synchronism with the clock signals or hunt excessively. The machine has an ultimate load capacity of $4 \mathrm{kw}$, and can take a suddenly applied load of $2 \mathrm{kw}$ without serious hunting.

\section{CONTENTS}

I. Introduction

II. Description of apparatus

1. Source of true 60 cycles........ 143

2. The synchroscope..... 145

3. The synchronous motor generator

III. Operation s.

1. Operation of the device as a whole......... 147

2. The accuracy of the machine...... 148

3. Protective devices.... 150

4. Possible improvements

IV. Application .

\section{INTRODUCTION}

Methods for accurately measuring time intervals are always of interest in laboratory work. This is particularly so in laboratories where such instruments as frequency meters and watthour meters are tested. The large number of observations necessary in work of this character, as well as the necessity of holding power or frequency constant during the measured time interval, makes it imperative that the time consumed by each individual observation be as short as is consistent with accuracy. The need thus arises for a timing device which will permit an accuracy in time measurements of at least 1 part in 10,000 over a reasonably short interval of time. Such a device should be capable of operating continuously and reliably over con- 
siderable intervals of time without attention. It should preferably have a sufficiently large load capacity to supply simultaneously a number of separate laboratories with time signals and to be reasonably unaffected by sudden large changes in load.

The nature of these requirements reduces considerably the number of types of devices available. The use of stop watches can be ruled out immediately both because of their uncertain starting and stopping error and because of their well-known fragility. ${ }^{1}$ For some time the timing device used in making watthour meter tests at the Bureau of Standards has been a 20-cycle, clock-driven, tuning fork. A contact on this fork is arranged to close an electrical circuit and thus operate a cycle counter. Such an arrangement has two serious disadvantages. Each failure of the fork to completely close the circuit, as a result of dirty or poorly adjusted contacts, introduces a direct error into the observations. In addition, only a small amount of power can be drawn through such a contact.

These limitations would be avoided by the use of a rotating device which is forced to maintain a rotary motion of constant angular velocity. This device could be used to drive an alternator for the production of alternating current of constant frequency. The use of rotating machinery maintained in synchronism with standard time signals from a tuning fork or similar device has two chief advantages. First, the relatively large amount of energy present as energy of rotation assures continuity of operation even though the control mechanism should momentarily fail to function. Second, it can be so arranged that the control mechanism, such as a tuning fork, is no longer forced to carry the entire operating current of the device.

Devices embodying some of these principles are on the market. None are available, however, which have the high load capacity which it was desired to obtain from the machine to be described here. A synchronous motor-generator set having an ultimate capacity of $4 \mathrm{kw}$ was, therefore, designed to operate in synchronism with time signals from a clock-controlled tuning fork. The principle upon which this machine operates is briefly as follows:

The rotor of a synchronous motor running synchronously from a supply line turns with respect to its d. c. magnetic field at an angular velocity determined solely by the frequency of the a. c. supply. If it is possible to rotate this d. c. magnetic field with respect to some stationary reference frame- that is, with respect to the stationary motor frame-the rotor of the synchronous motor will be forced to rotate with respect to this stationary frame at an angular velocity corresponding to the algebraic sum of the angular velocity determined by the a. c. supply frequency and the angular velocity of the d. c. magnetic field. In the machine described here the field coils of the synchronous motor are so wound and connected to an external commutator that a rotation of the commutator brushes produces a rotation of the magnetic field about the stationary motor frame. Means are then provided for rotating these commutator brushes, and hence the magnetic field of the motor, at an angular velocity directly proportional to the algebraic difference between true 60 cycles and the a. c. supply frequency. The rotor of the synchronous motor is thus forced to rotate, with respect to the stationary motor frame, at an angular

1 A. L. Ellis, The Limitations of the Stop Watch as a Precision Instrument, Trans. Am. Inst. Elec. Eng., vol., 40, pp. 479-502; 1921 . 
velocity directly proportional to true 60 cycles. The device thus consists of the following essential parts: A source of true 60 cycles, consisting in this case of two thyratron tubes operating in an inverter circuit excited by a clock-controlled tuning fork; a synchroscope or device for producing the proper rotation of the commutator brushes mentioned above; and a specially wound synchronous motor-generator set.

The use of such a rotating magnetic field was originally suggested by $\mathrm{H}$. B. Brooks, of this bureau, who had, many years ago, demonstrated experimentally the feasibility of such an arrangement. Most of the credit for the early development work in connection with the present apparatus should go to R. D. Wyckoff, formerly of this bureau. Mr. Wyckoff is responsible, in particular, for the design of the specially wound synchronous motor now in use. Mention should also be made of the contributions of V. E. Whitman, formerly of this

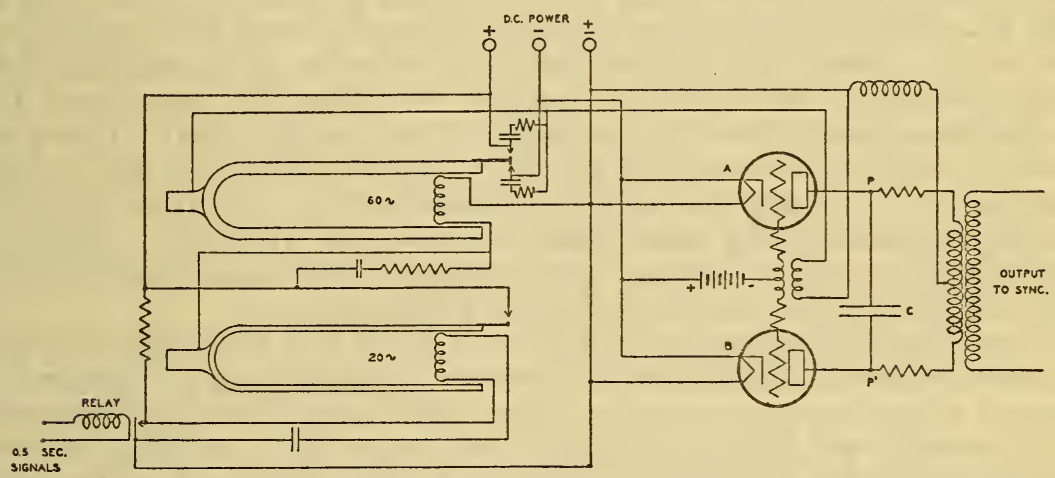

Frgdre 1.-Schematic diagram of the clock-controlled tuning-fork circuit and the thyratron inverter circuit

bureau, and others who have worked with the apparatus at various times.

\section{DESCRIPTION OF APPARATUS}

\section{SOURCE OF TRUE 60 CYCLES}

A chronometer made by Mercer, of London, serves as the primary time standard of this device. As certified by the time section of this bureau, this chronometer has a mean daily rate of -0.8 second at $20^{\circ} \mathrm{C}$. where the minus ( - ) sign indicates that the chronometer is gaining time. The pallet fork of this chronometer is equipped with a contact which can be used to actuate an external relay. A comparison of this contact against a standard half-second pendulum in the manner described by Moon ${ }^{2}$ indicated that this pallet fork could be relied upon to repeat its contact at half-second intervals to within one or two thousandths of a second.

The chronometer relay furnishes half-second impulses to the drive magnet of a 20 -cycle fork by means of the usual arrangement which utilizes a condenser discharge. Figure 1 is a schematic diagram of

${ }^{2}$ Charles Moon, A Precision Method of Calibrating a Tuning Fork by Comparison with a Pendulum, B. S. Jour. Research, vol. 4, pp. 213-219; 1930. 
the tuning-fork and thyratron circuits. The 60-cycle fork is driven in turn by impulses taken from a single-spring contact operated by the 20 -cycle fork as is indicated in the same figure.

The steel forks were obtained from the Cambridge Instrument Co. Unfortunately the addition of the spring contacts altered the period of each fork sufficiently to necessitate the addition of small tuning weights. This, however, is not a serious matter, since the forks are not depended upon to maintain their calibrations, but are forced to vibrate in synchronism with signals from the standard clock. For this reason it has not been found necessary to provide a constanttemperature inclosure for the forks.

The output of the 60-cycle fork is obtained from a double spring: contact on one prong of the fork. This contact vibrates between two stationary spring contacts which are insulated from each other and from the base and connected, respectively, to the $(+)$ and $(-)$ sides of a 230-volt d. c. power line as is indicated in Figure 1. One side of the 60-cycle fork output line is brought directly from the neutral post of the d. c. power line. The other is connected to the base of the fork which, through the vibrating contact, is alternately connected 115 volts above and below the potential of the neutral post. There results in the output circuit of the fork, which contains the grid transformer of the thyratron circuit, an alternating current of approximately square-topped wave form but 60-cycle frequency. Each of the fork contacts is bridged by a condenser with series resistance to quench sparking at the contacts in the usual manner.

The output of this 60-cycle fork excites the grid transformer of the thyratron circuit as is shown in Figure 1. Operating in an "inverter" circuit which has been described elsewhere ${ }^{3}$ these thyratrons generate in the output windings of the plate transformer an alternating current whose frequency is determined solely by the frequency of the grid excitation.

The operation of the inverter circuit may be briefly described in the following manner: A 60-cycle voltage derived from the tuning fork is supplied to the grids of the tubes. (See fig. 1.) The magnitude of this voltage is just sufficient to overcome the grid bias battery and cause operation of the tubes. Suppose now that the grid of tube $A$ has become positive, causing this tube to become conducting. A pulse of current flows through the plate transformer. The point $P$ is now at a more negative potential than point $P^{\prime}$ and the condenser $C$ receives a corresponding charge. On the next peak of the grid voltage wave the grid of tube $B$ becomes positive and tube $B$ becomes conducting. The condenser $C$ now discharges through the resistance and the transformer coils. If the resistances and capacitances have been properly chosen, the negative potential built up at $P$ during the finite duration of this discharge will be sufficient to make the plate of $A$ momentarily negative with respect to its cathode and thus stop the discharge through $A$. A pulse of current now flows through tube $B$, and through the transformer in a direction opposite to that of the first pulse. These operations repeat themselves as long as the alternating grid supply is maintained. It is obvious that the frequency of the current in the output transformer will always be identical with that of the grid supply. 
The current through the tuning-fork contacts when used in this circuit is of the order of 10 milliamperes. The average plate current through the thyratrons, FG-37, is of the order of 2.0 to 2.5 amperes. This current gives a drop of about 60 volts across the low-side coils of the plate transformer which is connected 360/60. This plate transformer supplies the rotor circuit of the synchroscope with an alternating current of true 60-cycle frequency.

\section{THE SYNCHROSCOPE}

The synchroscope is essentially a motor designed to operate at a speed and in a direction corresponding to the difference in frequency between the current supplied to its rotor and the current supplied to its stator system. Such a rotary synchroscope has been described in some detail by Lincoln. ${ }^{4}$ In the machine used here the stator is provided with a 3-phase, 4-pole, 110-volt, Y-connected, standard polyphase winding. The rotor contains a single-phase 4 -pole winding on salient poles. In its present application the rotor circuit is supplied with 60-cycle current from the thyratron circuit while the stator is supplied from the same 3-phase commercial power supply which is used to drive the synchronous motor. A rotation of the synchroscope rotor is thus obtained which corresponds both in magnitude and direction with the difference between the frequency of the commercial power supply and the true 60 cycles of the tuning-forkthyratron circuit. The brush rigging which supplies current to the d.c. field of the synchronous motor through its commutator, and thus produces a rotation of the motor field, is rigidly attached to the shaft of the synchroscope rotor.

The successful operation of the device as a whole depends largely upon the fidelity with which the synchroscope rotor can be made to follow the variations in frequency of commercial power. It thus becomes necessary to consider the equation for the torque acting on the synchroscope rotor and the equation of motion for the synchroscope rotor itself.

The current and torque equations and the equation of motion of the synchroscope rotor are given as equations (8), (9), and (11), respectively, in the Appendix. It is apparent from equations (8) and (9) that the component of torque tending to produce the desired rotation of the rotor; that is, $2 K A D \sin \left[\left(\omega_{2}-\omega_{1}\right) t-\beta-\theta_{1}\right]$, is produced by the interaction of the two currents flowing as the direct result of the applied rotor and stator voltages. The torque equation and the equation of motion indicate that the effect of the induced components of current in the rotor and stator circuits is to produce a double frequency disturbance in the motion of the synchroscope rotor. These induced components of current are, therefore, suppressed by increasing both the stator and rotor impedances. The stator voltage is raised to 480 volts and the stator impedance, per phase, is increased to approximately $800 \mathrm{ohms}$ by means of external choke coils. Similarly, the rotor voltage is raised to approximately 360 volts and the rotor impedance to approximately $700 \mathrm{ohms}$. These precautions reduce the induced-current components of equation (8) to magnitudes

4 P. M. Lincoln, Synchronism and Frequency Indication, J. Am. Inst. Elec. Eng., vol. 18, pp. 255270; 1901 . 
not more than 0.1 those of the main current components $E_{r} A \cos$ $\left(\omega_{1} t-\gamma\right)$ and $E_{s} D \cos \left(\omega_{2} t-\alpha-\zeta\right)$.

As an additional precaution the induced-current component $E_{s} C$ $\cos \left(\omega_{2} t-\beta-\alpha-\delta\right)$ is still further suppressed in the following manner: If the synchroscope rotor is stationary, the frequency of this induced component of rotor current will be $\omega_{2} / 2 \pi$, which is the same as the commercial frequency and which is the same as the frequency of the generator output when the motor is running synchronously from the line. If, however, the synchroscope is rotating at its proper velocity, $\left(\omega_{2}-\omega_{1}\right)$, the frequency of this component will be $\frac{\omega_{2}-\left(\omega_{2}-\omega_{1}\right)}{2 \pi}$ or $\omega_{1} / 2 \pi$. This again is the frequency of the generator output since under these conditions the motor will be running in synchronism with the tuning fork whose frequency is $\omega_{1} / 2 \pi$. It thus appears that under all steady operating conditions the frequency of the induced current component in the synchroscope rotor will be the same as the frequency of the generator output. A voltage may, therefore, be taken from the generator output having such a magnitude and phase that, when introduced into the rotor circuit of the synchroscope, it completely supresses the current component $E_{s} C \cos \left(\omega_{2} t-\beta-\alpha-\delta\right)$.

In addition to the double-frequency disturbance there are present in the motion of the synchroscope rotor, as is shown in equation (9), the damped free vibrations of the system acting as a torsion pendulum. If the motion of the synchroscope rotor under the influence of its main driving torque was strictly uniform, that is, if $\left(\omega_{2}-\omega_{1}\right)$ was constant with time, these free vibrations would be damped out quickly. Such, however, is not the case. The commercial frequency actually varies slightly but continually. As a result, the motion of the synchroscope rotor consists of a slow but irregular rotation, the rotor frequently coming to a complete stop. Some damping must, therefore, be present. It is also important that the free period of the system be large in comparison with the period of these variations of the line frequency and in comparison with the free period of the motorgenerator set (approximately 0.5 second). To this end an iron flywheel has been attached to the rotor shaft, giving the rotor system a moment of inertia of approximately $2.3 \times 10^{5} \mathrm{~g}-\mathrm{cm}^{2}$. With the maximum torques developed in the synchroscope, approximately $500 \mathrm{~g}-\mathrm{cm}$, the system has a minimum free period of about four seconds. This moment of inertia reduces to a negligible amount the response of the rotor system to the double-frequency components of torque. It is found, in addition, that the friction of the commutator brushes is sufficient to satisfactorily damp out the transient terms in the equation of motion of the synchroscope.

Under these conditions the synchroscope, once pulled into step, will satisfactorily operate over a frequency difference, $\left(\omega_{2}-\omega_{1}\right)$, of \pm 2.5 cycles. This gives an ample margin of safety since the commercial frequency in Washington is normally within a few tenths of a cycle of true 60 cycles.

\section{THE SYNCHRONOUS MOTOR-GENERATOR}

The synchronous motor now in use was obtained by modifying a 7.5-horsepower, 60-cycle, 3-phase, 440-volt induction motor which had a wound rotor. In its present application the motor action is inverted; 
that is, 3-phase a. c. power is supplied to the rotor, and the stator coils, which have been rewound and brought out to an external commutator, are excited with direct current for synchronous operation. The rotor winding is the original factory winding. From data obtained on the original stator winding when connected as a completely distributed 4-pole d. c. field, a new stator winding was designed to give normal excitation from a 120-volt d. c. supply. The final stator winding consists of 59 coils having 72 turns of No. 21 A. W. G. singlesilk-enamel wire per coil. The double-layer wave winding using diamond-shaped coils as ordinarily used in induction-motor stator windings was adopted. The coils were connected in the same manner as a d. c. motor armature having a 4-pole 2-circuit winding, and the leads brought out for connection to a 59-bar commutator. A rotation of the commutator brushes therefore produces an actual rotation of the d. c. magnetic field about the stator frame.

In its normal operation the motor is constantly subjected to torsional impulses and disturbances as a result of the continual shifting of its d. c. magnetic field about the stator frame. It is important, therefore, to have the machine heavily damped. For this reason the damping winding was made as heavy as the available space permitted. Reactors, each having an impedance of approximately 2 ohms at 60 cycles, have been placed in series with the rotor. Finally, the motor is run very much underexcited. It is possible to materially reduce the frequency of the hunting of the machine, but not necessarily the amplitude of the hunting, by the addition of a heavy flywheel to the rotor shaft. The presence of such a flywheel makes it necessary to provide some external means; that is, other than the torque of the currents induced in the damping windings, of bringing the motor up to synchronous speed. The machine is, therefore, not provided with a flywheel.

A 3-phase, 4-kw, 60-cycle generator is directly coupled to the shaft of the synchronous motor. This generator constitutes a source of true 60 cycles which can be used to operate cycle counters, synchronous timers, small synchronous motors, or other similar devices. In addition to its a. c. windings, the generator is provided with a commutator and d. c. windings of sufficient capacity to provide the excitation of the generator field and synchronous motor field should this become desirable.

Figure 2 is a diagrammatic sketch of the connections used in the set-up. Figures 3 and 4, respectively, are photographs of the assembled tuning-fork-thyratron apparatus and the motor-generator set itself.

\section{OPERATION}

\section{OPERATION OF THE DEVICE AS A WHOLE}

The operation of the device as a whole proceeds as follows: The synchronous motor is brought up to speed with the induction-motor torque of its damping winding and is synchronized with the commercial a. c. power line. The synchroscope stator is excited from the same commercial a. c. power line. The generator field is excited and a voltage is taken from the generator output having the proper phase and magnitude to exactly neutralize the induced voltage component in the rotor circuit of the synchroscope. The tuning fork and thyratron circuits are then put into operation and the output of the thyratron 
circuit introduced into the synchroscope rotor. The synchroscope rotor then rotates, and with it the synchronous motor field. Under these conditions, as has been explained above, the synchronous motor is forced to rotate at a speed corresponding to the frequency of the tuning-fork supply, which is 60 cycles. The output of the generator may now be used for any purposes which call for a current of true 60 cycles, averaged over as much as a few seconds.

Experiment has shown that the machine can safely take a suddenly applied load of as much as $2 \mathrm{kw}$ without excessive hunting.

\section{THE ACCURACY OF THE MACHINE}

In its use as a timing device the apparatus is subject to two major sources of error. First, even if the motor could be so adjusted as to.

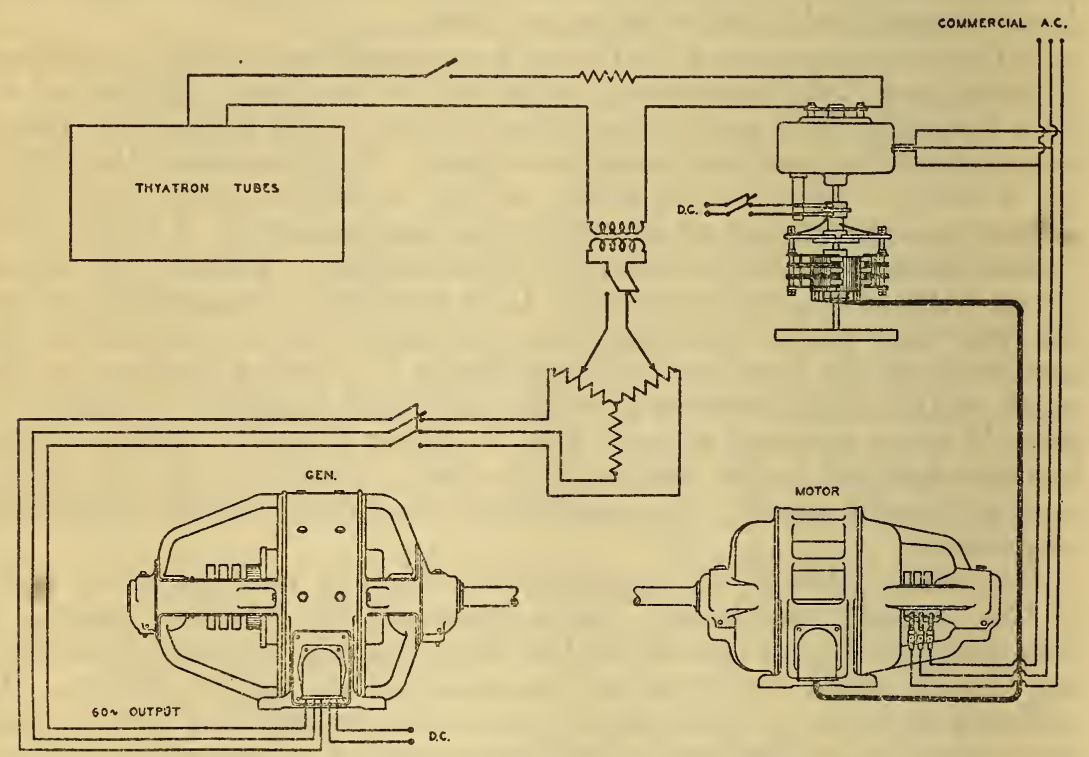

FIGURE 2.-Schematic wiring diagram of the clock-controlled synchronous motor-generator set

maintain precisely and without hunting its exact synchronism with the 60 -cycle fork, and thus with the signals from the chronometer, its time signals could never be more reliable than those of the chronometer. The daily rate of this chronometer is, as has been stated, of the order of -0.8 second a day. This amounts to a systematic error of about 1 part in 100,000, and is therefore entirely negligible for all the purposes for which this generator will be used. Second, the motor does not actually, from instant to instant, maintain exact synchronism with the 60-cycle fork, but is continually hunting about this synchronous position. To obtain a quantitative estimate of the error in troduced by this hunting, the following experiment was performed:

A stroboscope was set up which consisted of a neon lamp lighted by the 60-cycle fork output and viewed through a slotted disk on the end of the generator shaft. The hunting of the machine then became apparent as an oscillation through a small angle of the resulting bright 
B. S. Journal of Research, RP406

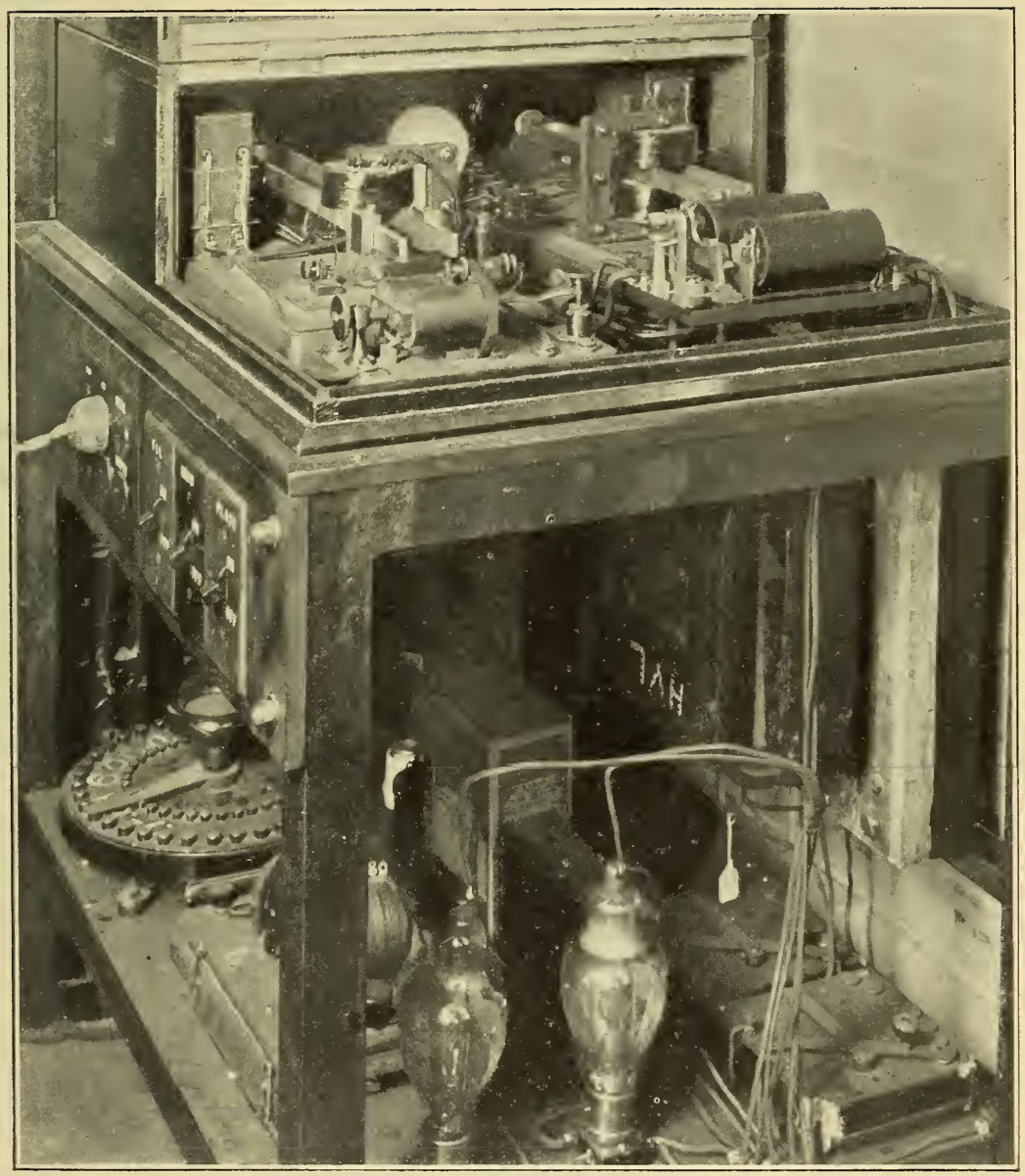

FIGURE 3.-Photograph of the assembled tuning-fork-thyratron apparatus 
B. S. Journal of Research, RP406

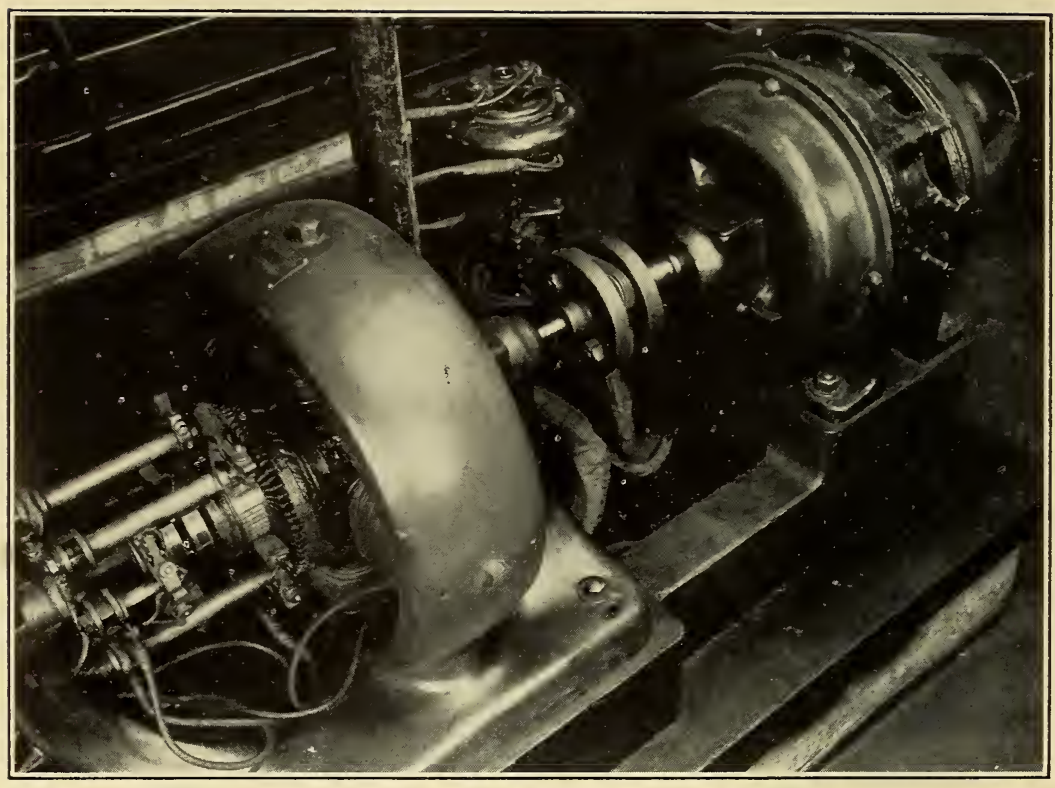

FIGURE 4.-Photograph of the complete clock-controlled motor-generator set and synchroscope 
slit image. The instantaneous position could be determined with a protractor placed behind the slotted disk. The neon lamp circuit was arranged to be closed approximately once every three seconds, and the instantaneous position of the generator shaft was read and recorded for each flash of the neon lamp. Over 1,300 individual observations were made, spread over a total interval of approximately five hours. The resulting observations were averaged and plotted, after the fashion of an ordinary error function, as probability against departure from the mean. These data are plotted in Figure 5 in which the deviation from the mean in mechanical degrees has been reduced to the resulting error in time, $\Delta t$, in thousandths of a second. Since the generator shaft is turning at the rate of 10,800 mechanical de-

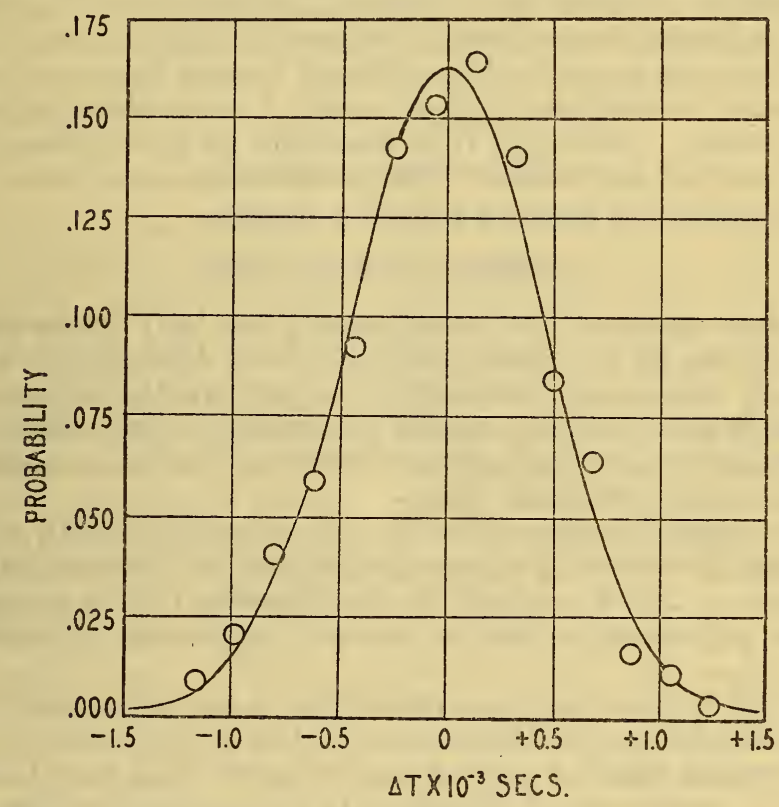

FIGURE 5.-Probability of a given error in time measurement introduced by the random hunting of the machine

O, observed points; - , plotted from the equation $y=0.162 e^{-(1.56 \Delta t)^{2}} ; \Delta t$ in thousandths of a second.

grees per second, 10.8 mechanical degrees correspond to 0.001 second. The solid curve in Figure 5 represents the probability equation

where

$$
y=0.162 e^{-(1.56 \Delta t)^{2}}
$$

$y$ is the probability.

$\Delta t$ is the amount by which the machine is away from its mean position expressed in thousandths of a second.

The constant for this curve was obtained from the experimental data by means of Peter's rule. The probable error of the machine, computed from this equation, is \pm 0.0003 second. Similarly, it can be verified that the probability that the machine will be within \pm 0.002 second of its mean position at any instant is 0.99998. 
It is to be noted that this error is a truly random one and is not cumulative. When time intervals of any appreciable length are being measured, say 50 or 100 seconds, this random error becomes entirely negligible.

A safety device, to be described in the next section, is provided which definitely shuts down the machine if, for any reason, this instantaneous hunting error amounts to more than a predetermined amount, say, \pm 0.004 second.

Since the free period of the motor-generator set is fairly short, being something less than 1 second, the variations in angular position taking place as a result of the hunting of the machine occur with considerable rapidity. Consequently the variations in instantaneous frequency are appreciable. As read on a General Electric type P3 tuned-circuit frequency indicator, the apparent frequency variations are of the order of \pm 0.1 cycle per second. It is difficult to say how much of this is true frequency variation and how much is over-shooting of the instrument needle. Although it is desirable to have these frequency variations as small as possible, their presence is no particular handicap when the machine is used as a timing device.

\section{PROTECTIVE DEVICES}

The various parts of the motor circuit are fully protected against accidental abuse by the usual safety devices; that is, the a. c. power line is fused, and the motor starting box is provided with an overload relay which guards against excessive current in the motor armature and a no-voltage relay which opens the motor circuit in case the voltage falls below a predetermined value.

The d. c. supply to the 60-cycle fork is supplied with a 2-ampere relay to clear the circuit if an arc should develop between the 60 -cycle fork contacts. The d.c. supply to the thyratron plate circuit is supplied with a 6-ampere fuse to prevent damaging currents in this circuit.

In addition to these devices, protection is needed against the possibility that the forks, the thyratrons, or the synchroscope may fail to function, or that the d. c. power to the motor field may fail. In the first case the motor would continue to run in synchronism with the line frequency without damage to itself, but as a timing device it would no longer be reliable. In the second case the motor would continue to run as an induction motor on the torque arising from the damping windings. As a timing device it would no longer be accurate, and the motor itself would be subject to injury, because of the small load capacity of the damping winding. Some precaution must also be taken against excessive hunting on the part of the motor.

To take care of these possibilities a differential relay was constructed. This relay is mechanically similar to an ordinary telegraph relay, except that the core and armature are laminated. One of the two coils on this relay is excited from the true 60-cycle source in parallel with the synchroscope rotor. The other coil on the relay is excited from the generator output. Each of these coils contains 7,000 turns of No. 33 A. W. G. silk-enamel wire and draws a current of approxinately 75 milliamperes. When the generator is running in synchronism with the fork, these voltages are so chosen and adjusted that the magnetizing forces from the two coils aid each other. The spring tension on the armature of the relay is then adjusted until the re- 
sultant force from the two coils is just sufficient to hold the relay armature in place. If, for any reason, either of these two sources of excitation should fail, or if, for any reason, the generator voltage should swing out of phase with respect to the fork voltage, as by excessive hunting, by more than a predetermined amount, the relay armature will be released. The releasing of the relay armature closes a by-pass which short-circuits the magnet coils of the no-voltage relay of the starting box and shuts down the synchronous motor. The relay can be adjusted conveniently to release on a phase displacement of the motor of about 40 mechanical degrees from its mean position. This corresponds to about \pm 0.004 second or about a quarter of a cycle. This adjustment allows the ordinary and unavoidable hunting of the machine to take place and yet guards definitely against an error amounting to more than about \pm 0.004 second. The observer is also assured that the machine is running properly and in synchronism as long as voltage is obtained on the output lines.

\section{POSSIBLE IMPROVEMENTS}

Several improvements might be made if the construction of a similar piece of apparatus should ever be considered. A more heavily damped motor would do much to eliminate the frequency variations now taking place. In this connection the mercury flywheel suggests itself as a possibility. With a critically damped motor the accuracy of the machine would be limited solely by the accuracy of the chronometer, the finite subdivision of the motor field, and the ability of the synchroscope to follow faithfully and instantly the variations in the frequency of commercial power. In the present apparatus the effect of the finite subdivision of the motor field has been entirely negligible. In a more accurately controlled machine, however, its effect might become appreciable. Without doubt a more efficient synchroscope could be designed for operation under the conditions imposed by the present application. In this connection one of the small indicator motors on the market, such as the Selsyn motor, is worthy of consideration. A preliminary investigation of one of these motors indicated that it would develop a very satisfactory amount of torque when substituted for the present synchroscope.

The spring contacts now in use on the tuning forks were installed at a time when it was necessary to draw a rather heavy current through these contacts. The thyratron grid circuit requires so little current that this particular difficulty is now avoided. It should, therefore, be possible to use platinum wire contacts dipping in mercury wells. Such contacts have much less effect on the period of the fork than the ones now in use.

\section{APPLICATIONS}

The output of the machine described here may be used to operate cycle counters, synchronous timers, or any of the other electrical timing devices on the market. A synchronous clock or timer operating from the a. c. output of this machine can be relied upon to keep the time of the master chronometer over a period of as much as 4 or 5 seconds or longer with a probable error of \pm 0.0003 second and a maximum error of \pm 0.004 second. These errors are far smaller then can be detected on commercial synchronous timers, most of which can be read to only 0.01 second. The output of the machine 
may also be used to operate small synchronous motors driving chronographs or other synchronous machinery whose average speed, taken over a period of 4 or 5 seconds, must be constant to a high degree of precision.

The unique characteristic of the machine is its large load capacity. Although possessing the inherent accuracy of the master chronometer, the machine can take a suddenly applied load of $2 \mathrm{kw}$ and is capable of carrying a continuous load of $4 \mathrm{kw}$ without injury to itself. There are no serious limitations on the size of such a machine. The high accuracy of the machine, its large load capacity, and the automatic protective devices which can be used with it, make it particularly adapted to installations where a large number of laboratories must be supplied with time signals.

This apparatus was primarily designed for, and finds its greatest use in, the measurement of short-time intervals in the routine calibration of commercial instruments, such as watthour meters and frequency meters. The necessity of holding watts or frequency constant during the time interval measured makes it imperative that that time interval be as short as is consistent with accuracy. Commercial impulse counters and synchronous timers are used to measure these intervals, the accuracy of the measurement being limited at present solely by the accuracy of the recording device. With such instruments it is possible to measure time intervals of 10 seconds with an accuracy of 1 part in 1,000 and intervals of 100 seconds with an accuracy of 1 part in 10,000. These limits represent a marked improvement over the accuracy obtained when synchronous timers are used directly on a commercial line. It is well known ${ }^{5}$ that even in systems where the frequency is closely controlled and averages 60 cycles to a high degree of precision over long intervals of time, the instantaneous frequency can not be relied upon to more than about 1 part in 600 over short intervals of time. The accuracy of the present machine is such that it would justify the use of a more precise instrument for recording these time intervals than is now commercially obtainable.

In calibrating frequency meters in the neighborhood of 60 cycles, it is possible to use an impulse counter to count the electrical beats between the unknown frequency and the true 60 cycles of this generator and measure the corresponding time interval. This method leads to a considerable saving in time and increase in accuracy in the measurement as compared with the usual method of counting cycles directly and measuring the corresponding time interval.

The installation of a 1,000-cycle generator on the shaft of this motor-generator set would furnish a convenient source of 1,000cycle current, of controlled frequency, for use in routine bridge measurements about the laboratory.

In addition to these applications, the apparatus should find use in the Bureau of Standards in driving the small synchronous motors used in timing exposures in photographic research, and in measuring short-time intervals in X-ray and radio-activity work.

A device similar in principle to that described here may find use in the maintenance of isochronism in small motors such as is necessary

5J. M. Adams, Time Measuring by Commercial Alternating Current with Controlled Frequency, J. Opt. Soc. Am., vol. 19, pp. 384-386, 1929. 
in the operation of machine telegraph systems. ${ }^{6}$ A similar application may be found in the operation of radio direction-finding apparatus. It is stated ${ }^{7}$ that in such installations it is not only necessary to operate two stations in exact synchronism, but also to rotate the loop antennas at a velocity of 1 revolution per minute with such uniformity that the loop shall never be more than 1 degree from its proper position. With a synchronous motor running from the machine described here and driving a suitable reduction gear to give 1 revolution per minute, one could be assured that the final rotating member would never be more than \pm 0.02 degree from its proper position.

\section{APPENDIX. DERIVATION OF THE EQUATION OF MOTION OF THE SYNCHROSCOPE}

Consider a synchroscope with a symmetrical 3-phase stator and a single-phase rotor. The position of the rotor in space is defined by the angle $\beta$, measured from an initial line determined by the position of maximum mutual inductance between the rotor coil and stator coil $a$. Since the mechanical frequency of rotation of the synchroscope rotor will always be very small compared to the frequency of alternation of the supply currents, we shall neglect the currents induced by this mechanical rotation. That is to say, we shall neglect terms of the order $d \beta / d t$. We thus treat $M$, the mutual inductance between the rotor and stator circuits, as a function of $\beta$ and hence of $t$ (since $\beta$ is the explicit function of $i$ which we wish to find), but neglect terms of the order $d M / d t$. We assume that the mutual inductances between the rotor coil and the successive coils of the 3-phase stator system can be expressed as sinusoidal functions of the angle $\beta$.

With these assumptions in mind, let the rotor circuit alone be excited, the stator coils being closed through circuits of normal impedance, but with no applied emf. The equations for the resulting instantaneous voltages are

$$
\begin{gathered}
\frac{d}{d t}\left[L i_{\tau}+M_{a} i_{a}+M_{b} i_{b}+M_{c} i_{c}\right]+R i_{\tau}=e_{\tau} \\
\frac{d}{d t}\left[M_{k} i_{r}+N i_{k}\right]+S i_{k}=0, k=a, b, c .
\end{gathered}
$$

where

$e_{r}$ is the instantaneous applied rotor voltage.

$i_{\tau}$ is the instantaneous rotor current.

$i_{a}, i_{b}$, and $i_{c}$ are the instantaneous currents in stator phases $a, b$, and $c$, respectively.

$R$ is the resistance of the rotor circuit in ohms.

$S$ is the resistance of each stator phase in ohms.

$L$ is the self-inductance of the rotor circuit in henries.

$N$ is the self-inductance of each stator phase in henries.

$M_{a}, M_{b}$, and $M_{c}$ are the mutual inductances, in henries, between the rotor coil and stator coils $a, b$, and $c$, respectively.

\footnotetext{
${ }^{6}$ H. H. Harrison, Developments in Machine Telegraph Systems and Methods of Operation, J. Inst. Elec. Eng. (London), vol. 69, pp. 1369-1453: 1930.

7 N. F. S. Hecht and D. P. Alexander, A Device for Maintaining Isochronism in Low-Power Electric Motors, J. Inst. Eloc. Eng. (London), vol. 69, pp. 83-88; 1930.
} 
Now make the usual substitutions defined by the real parts of the following exponentials:

$$
\begin{aligned}
& e_{\tau}=E_{\tau} e^{j \omega_{1} t} \\
& i_{\tau}=G e^{j \omega_{1} t} \\
& i_{a}=H \cos \beta e^{j \omega_{1} t} \\
& i_{b}=H \cos \left(\beta-\frac{2 \pi}{3}\right) e^{j \omega_{1} t} \\
& i_{c}=H \cos \left(\beta-\frac{4 \pi}{3}\right) e^{j \omega_{1} t}
\end{aligned}
$$

where

$E_{\tau}$ is the maximum value of the applied rotor voltage.

$G$ is the maximum value of the current in the rotor circuit.

$H$ is the maximum value of the current in each stator coil.

$j$ is the imaginary operator $\sqrt{-1}$.

$\omega_{1}$ is $2 \pi \times$ the frequency of the applied rotor voltage. Finally, assume

$$
\begin{aligned}
& M_{a}=M_{o} \cos \beta \\
& M_{b}=M_{o} \cos (\beta-2 \pi / 3) \\
& M_{c}=M_{o} \cos (\beta-4 \pi / 3)
\end{aligned}
$$

Substitution of equations (2) and (3) in equation (1) leads to the following linear equations which serve to determine $G$ and $H$ :

$$
\begin{gathered}
\left(R+j \omega_{1} L\right) G+\frac{3}{2}\left(j \omega_{1} M_{0}\right) H=E_{r} \\
j \omega_{1} M_{0} G+\left(S+j \omega_{1} N\right) H=0
\end{gathered}
$$

The complex values for $G$ and $H$ obtained from equations (4) are to be substituted in equations (2) and the real parts of the resulting currents retained.

These operations lead to current equations of the following forms, for the case of rotor excitation alone:

$$
\begin{aligned}
& i_{r}=E_{r} A \cos \left(\omega_{1} t-\gamma\right) \\
& i_{a}=E_{r} B \cos \beta \cos \left(\omega_{1} t-\varepsilon\right) \\
& i_{b}=E_{r} B \cos \left(\beta-\frac{2 \pi}{3}\right) \cos \left(\omega_{1} t-\varepsilon-\frac{2 \pi}{3}\right) \\
& i_{c}=E_{r} B \cos \left(\beta-\frac{4 \pi}{3}\right) \cos \left(\omega_{1} t-\varepsilon-\frac{4 \pi}{3}\right)
\end{aligned}
$$

where

$A$ and $B$ are constants, determined by the circuit constants, having the dimensions of admittance.

$\gamma$ and $\varepsilon$ are phase angles determined by the circuit constants.

Consider now the case in which the stator coils are excited from a 3 -phase source, the rotor being closed through a circuit of normal 
impedance, but with no applied emf. The equations for the resulting instantaneous voltages are-

$$
\begin{gathered}
\frac{d}{d t}\left[L i^{\prime}{ }_{r}+M_{a} i^{\prime}{ }_{a}+M_{b} i^{\prime}{ }_{b}+M_{c} i^{\prime}{ }_{c}\right]+R i^{\prime}{ }_{r}=0 \\
\frac{d}{d t}\left[M_{k} i^{\prime}{ }_{r}+N i^{\prime}{ }_{k}\right]+S i^{\prime}{ }_{k}=E_{k}, k=a, b, c
\end{gathered}
$$

where the symbols have the same meanings as in equation (1), the currents in this case being distinguished from those in equation (1) by primes.

Following a procedure analogous to that used in the solution of equation (1), we obtain for the currents, equations of the following forms:

$$
\begin{aligned}
& i_{r}^{\prime}=E_{s} C \cos \left(\omega_{2} t-\alpha-\beta-\delta\right) \\
& i^{\prime}{ }_{a}=E_{s} D \cos \left(\omega_{2} t-\alpha-\zeta\right)+E_{s} F \cos \beta \cos \left(\omega_{2} t-\alpha-\beta-\eta\right) \\
& \begin{array}{r}
i^{\prime}{ }_{b}=E_{s} D \cos \left(\omega_{2} t-\alpha-\zeta-\frac{2 \pi}{3}\right)+E_{s} F \cos \left(\beta-\frac{2 \pi}{3}\right) \cos \left(\omega_{2} t-\alpha-\beta\right. \\
\left.\quad-\eta-\frac{2 \pi}{3}\right)
\end{array} \\
& \begin{array}{r}
i^{\prime}{ }_{c}=E_{s} D \cos \left(\omega_{2} t-\alpha-\zeta-\frac{4 \pi}{3}\right)+E_{s} F \cos \left(\beta-\frac{4 \pi}{3}\right) \cos \left(\omega_{2} t-\alpha-\beta\right. \\
\left.-\eta-\frac{4 \pi}{3}\right)
\end{array}
\end{aligned}
$$

where

$E_{s}$ is the maximum value of the applied stator voltage.

$C, D$, and $F$ are constants determined by the circuit constants and having the dimensions of admittance.

$\delta, \zeta$, and $\eta$ are phase angles determined by the circuit constants. $\omega_{2}$ is $2 \pi \times$ the frequency of the applied stator voltage.

$\alpha$ is the initial phase angle existing between the applied rotor and stator voltages.

Since under normal operating conditions both stator and rotor circuits are excited from separate sources, we obtain the complete expressions for the currents flowing in these circuits under normal operating conditions by adding equations (5) and (7).

$I_{r}=E_{\tau} A \cos \left(\omega_{1} t-\gamma\right)+E_{s} C \cos \left(\omega_{2} t-\alpha-\beta-\delta\right)$

$I_{a}=E_{r} B \cos \beta \cos \left(\omega_{1} t-\varepsilon\right)+E_{s} D \cos \left(\omega_{2} t-\alpha-\zeta\right)+E_{s} F \cos \beta \cos$ $\left(\omega_{2} t-\alpha-\beta-\eta\right)$

$$
\begin{gathered}
I_{b}=E_{\tau} B \cos \left(\beta-\frac{2 \pi}{3}\right) \cos \left(\omega_{1} t-\varepsilon-\frac{2 \pi}{3}\right)+E_{s} D \cos \left(\omega_{2} t-\alpha-\left\{-\frac{2 \pi}{3}\right)\right. \\
+E_{s} F \cos \left(\beta-\frac{2 \pi}{3}\right) \cos \left(\omega_{2} t-\alpha-\beta-\eta-\frac{2 \pi}{3}\right) \\
I_{c}=E_{\tau} B \cos \left(\beta-\frac{4 \pi}{3}\right) \cos \left(\omega_{1} t-\varepsilon-\frac{4 \pi}{3}\right)+E_{s} D \cos \left(\omega_{2} t-\alpha-\zeta-\frac{4 \pi}{3}\right) \\
+E_{s} F \cos \left(\beta-\frac{4 \pi}{3}\right) \cos \left(\omega_{2} t-\alpha-\beta-\eta-\frac{4 \pi}{3}\right)
\end{gathered}
$$


The various current components may be identified in the following manner: The components $E_{r} A \cos \left(\omega_{1} t-\gamma\right)$ and $E_{s} D \cos \left(\omega_{2} t-\alpha-\zeta\right)$, etc., represent currents flowing in the rotor and stator circuits as the direct result of the applied rotor and stator voltages, respectively. They may be referred to as the "main" rotor and stator currents. The component $E_{s} C \cos \left(\omega_{2} t-\alpha-\beta-\delta\right)$ represents a current induced in the rotor circuit by the components of stator current of frequency $\omega_{2}$. Similarly, the components of stator current $E_{\tau} B \cos \beta \cos$ $\left(\omega_{1} t-\varepsilon\right)$, etc., represent currents induced in the stator system by the main component of rotor current. The components of stator current $E_{s} F \cos \beta \cos \left(\omega_{2} t-\alpha-\beta-\eta\right)$, etc., represent currents induced in the stator system by the component of rotor current having a frequency $\omega_{2}$; that is, by the "induced" rotor current. We may, therefore, speak of these last three current components as "induced" currents.

The torque is obtained in the usual manner by forming the products

$$
\mathrm{T}=\Sigma I_{r} I_{s} d M_{s} / d \beta, s=a, b, c
$$

Products of sines are expanded into sums and differences of the corresponding angles and the sums, representing high frequency torques, are discarded. The resulting torque equation is of the following form:

$$
\begin{aligned}
\mathrm{T} & =K\left[2 A D \sin \left\{\left(\omega_{2}-\omega_{1}\right) t-\beta-\theta_{1}\right\}-A F \sin \left\{\left(\omega_{2}-\omega_{1}\right) t+\beta-\theta_{2}\right\}\right. \\
& -B C \sin \left\{\left(\omega_{2}-\omega_{1}\right) t-3 \beta-\theta_{3}\right\}-\frac{E_{r}}{E_{s}} A B \sin \left\{2 \beta-\theta_{4}\right\} \\
& \left.-\frac{E_{s}}{E_{\tau}} C F \sin \left\{2 \beta-\theta_{5}\right\}+2 \frac{E_{s}}{E_{\tau}} C D \sin \theta_{6}\right]
\end{aligned}
$$

where

$K$ is a constant having the dimensions of (voltage) ${ }^{2}$
$\theta_{1}, \theta_{2}, \theta_{3}, \theta_{4}, \theta_{5}$, and $\theta_{6}$ are constant phase angles.

As has been mentioned in the discussion of the synchroscope, the rotor and stator impedances have been increased to such an extent that the admittances $C, D$, and $F$ are approximately 0.1 the magnitude of the admittances $A$ and $D$, while the voltages $E_{r}$ and $\mathrm{E}_{s}$ are of the same order of magnitude. The last five terms of the torque equation will, therefore, be small in comparison with the first.

Let us now set up the equation of motion of the synchroscope rotor

$$
\begin{aligned}
& I d^{2} \beta / d t^{2}+k d \beta / d t=A_{1} \sin \left\{\left(\omega_{2}-\omega_{1}\right) t-\beta-\theta_{1}\right\}+A_{2} \sin \left\{\left(\omega_{2}-\omega_{1}\right) t+\beta-\theta_{2}\right\} \\
& \quad+A_{3} \sin \left\{\left(\omega_{2}-\omega_{1}\right) t-3 \beta-\theta_{3}\right\}+A_{4} \sin \left\{2 \beta-\theta^{\prime}{ }_{4}\right\}-A_{5}
\end{aligned}
$$

$\beta$ is the angle defining the position of the rotor in space as previously defined

$I$ is the moment of inertia of the rotor system

$k$ is the coefficient of damping of the system.

The other abbreviations made in the torque equation are obvious. It should be remembered that $A_{2}, A_{3}, A_{4}$, and $A_{5}$ are small compared with $A_{1}$.

We obtain an approximate solution, $\beta_{0}$, of equation (10) by neglecting the last four terms of the torque expression, and assuming that $\beta$ is never far from its synchronous value $\left(\omega_{2}-\omega_{1}\right) t$. Equation (10) then readily reduces to the linear form with constant coefficients 
arising from the forced vibrations of a harmonic oscillator. If $\beta_{0}$ is approximately $\left(\omega_{2}-\omega_{1}\right) t$ it is apparent that the last four terms in the torque expression in equation (10) reduce approximately to torques having a frequency $2\left(\omega_{2}-\omega_{1}\right)$. We find the effect of these disturbing double-frequency torques to a first approximation by assuming

$$
\beta=\beta_{0}+\Delta \beta
$$

substituting in equation (10), neglecting products and squares small quantities, and solving for $\Delta \beta$.

These operations lead to a solution for $\beta$ of the following form:

$$
\begin{gathered}
\beta=\left(\omega_{2}-\omega_{1}\right) t-\phi \\
+e^{-\frac{k t}{2 I}\left[C_{0} \cos \left(2 \pi \nu_{0} t-\delta_{0}\right)+C_{1} \cos \left(2 \pi \nu_{1} t-\delta_{1}\right)\right]} \\
+C_{2} \sin \left[2\left(\omega_{2}-\omega_{1}\right) t-\delta_{2}\right]
\end{gathered}
$$

where

$$
\begin{aligned}
2 \pi \nu_{0} & =\frac{\left[4 I A_{1}-k^{2}\right]^{1 / 2}}{2 I} \\
2 \pi \nu_{1} & =\frac{\left[4 I A_{1} \cos \frac{k\left(\omega_{2}-\omega_{1}\right)}{A_{7}}-k_{2}\right]^{1 / 2}}{2 I}
\end{aligned}
$$

$k^{2}$ is less than $4 I A$

$\mathrm{C}_{0}$ and $\mathrm{C}_{1}$ are amplitude factors depending upon the initial conditions

$C_{2}$ is an amplitude factor depending upon the magnitude of the disturbing torque, upon $1 / I$ and $1 / k$.

$\phi, \delta_{0}, \delta_{1}$, and $\delta_{2}$ are constant phase angles.

The motion of the synchroscope rotor, therefore, consists of a steady rotation at an angular velocity $\left(\omega_{2}-\omega_{1}\right)$, damped free vibrations of the system acting as a torsion pendulum, and undamped forced vibrations of a frequency $2\left(\omega_{2}-\omega_{1}\right)$.

Washington, November 11, 1931. 